\title{
Verification of object-oriented programs with invariants
}

\author{
Mike Barnett, Robert DeLine, Manuel Fähndrich, K. Rustan M. Leino, \\ Wolfram Schulte \\ Microsoft Research, Redmond, WA, USA
}

An object invariant defines what it means for an object's data to be in a consistent state. Object invariants are central to the design and correctness of objectoriented programs. This paper defines a programming methodology for using object invariants. The methodology, which enriches a program's state space to express when each object invariant holds, deals with owned object components, ownership transfer, and subclassing, and is expressive enough to allow many interesting object-oriented programs to be specified and verified. Lending itself to sound modular verification, the methodology also provides a solution to the problem of determining what state a method is allowed to modify.

\section{INTRODUCTION}

Writing and maintaining software is difficult and error prone, in part because it requires coping with many details. Mechanical programming tools can relieve some of this burden. For example, an important and pervasive tool is the type checker, which allows the programmer to describe in broad-brush terms the set of values each program variable can take. Using these descriptions, the type checker mechanically checks all reads and writes of program variables to ensure that no variable takes on a forbidden value. The type checker is usually built into the compiler, which also checks other details. For example, the compiler may check that every variable use is preceded by an assignment, that any read-only variable is not changed after its initial assignment, or that variables declared in certain scopes or with certain access modifiers are not referenced from inappropriate places. These successful detail management techniques have in common that the programmer formulates the condition that is supposed to hold and leaves the details of enforcing the condition to a mechanical tool.

In this paper, we consider object-oriented programs and focus on object invariants. An object invariant specifies a relation on an object's data that the programmer intends for to hold. Using object invariants, one can detect or prevent data corruption errors and other misuse of the data. Ultimately, we are interested in leaving the detail management of object invariants to a mechanical tool, but doing so requires that we first determine a good methodology for using object invariants.

The idea that objects, in their steady states, satisfy certain data invariants goes back 
at least to Hoare's 1972 paper on data-representation correctness [19]. Putting this idea into practice, Eiffel [33] is a language that has constructs for specifying object invariants and whose familiar "design by contract" design methodology has been used for almost two decades. These seminal systems, however, are not without their limits. For example, Hoare had considered an object model that does not include the useful references of modern object-oriented languages. Eiffel checks object invariants dynamically (that is, at run-time), typically on exit from public methods. With a good test suite, these dynamic checks catch many errors, but, as we shall see later, this approach does not always permit a programmer to assume an object's data to be consistent on entry to a public method. In fact, we know of only one previous methodology for a modern object-oriented language that provides useful guarantees about when object invariants hold, namely, Peter Müller's $\mathrm{PhD}$ thesis [35]. Müller's methodology builds on top of a special type system, the universe type system, that captures the hierarchy of layered abstractions commonly employed by well-designed programs. An object at one level of this hierarchy is said to own the objects in the level below. In the methodology, whether the invariant of an object holds is modeled by a boolean function on the object's data.

In this paper, we develop a methodology for reasoning about object invariants. Ultimately, we're interested in static verification of programs, but our methodology can also be used with dynamic checking. Our methodology leverages a program's hierarchy of abstractions, but does not use a type system for this purpose. Instead, the methodology tracks ownership relations more precisely and permits ownership transfer. Also, instead of using an abstract variable that, as a function of the object's data, models whether or not an object invariant actually holds (like in Müller's work and in the methodology of ESC/Modula-3 [28]), our methodology uses an independent variable that indicates whether the object invariant is known to hold. This opens the possibility of precisely formulating what program invariants the methodology guarantees, conditions that hold of a program in every reachable state. Finally, recognizing that the declarations of an object's data are divided up along a subclass hierarchy, our methodology allows separate reasoning at the level of each subclass.

In the next section, we motivate a central design decision in our methodology. The subsequent sections build up to our full methodology: Section 3 explains the basic idea of our methodology by considering individual objects; Section 4 adapts the basic idea to accommodate aggregate objects; and Section 5 makes the model more detailed to support subclasses. Section 6 considers the specification of methods in our methodology, where we will also provide a new solution to the notorious problem of how to specify which pieces of the program state a method may modify. We give some additional examples in Section 7 and then end the paper by describing more related work and giving our conclusions.

\section{OBJECT INVARIANTS AND INFORMATION HIDING}

There is a tension between object invariants and information hiding that has led us to an important design decision in our methodology: to explicitly represent whether an object 
a) class $T\{$

private $x, y$ : int ;

invariant $0 \leqslant x<y$;

public $T($ )

\{

\}

$x:=0 ; y:=1 ;$

public method $M()$

modifies $x, y$;

\{

assert $y-x \geqslant 0$;

$x:=x+3$;

$y:=4 * y$;

\}

\} b) class $T\{$

private $x, y$ : int ;

public $T($ )

ensures $0 \leqslant x<y$;

\{

$x:=0 ; y:=1 ;$

\}

public method $M()$

requires $0 \leqslant x<y$;

modifies $x, y$;

ensures $0 \leqslant x<y$;

\{

assert $y-x \geqslant 0$;

$x:=x+3$;

$y:=4 * y ;$

\}

Figure 1: (a) A simple program with an object invariant and routine specifications. (b) The same program, where the object invariant has been changed into various pre- and postconditions.

invariant is known to hold, and to expose this information in specifications. To explain the rationale behind our design, we start by considering a popular-but, as we shall see, problematic_-view of object invariants.

Figure 1(a) shows a simple program consisting of a class $T$ with two data fields, $x$ and $y$, a constructor, and one method, $M$. The program declares the object invariant $0 \leqslant x<y$. Each routine (method or constructor) is given a specification that spells out the contract between its callers and its implementation. A routine specification consists of three parts: a precondition, which describes the states in which a caller is allowed to call the routine; a postcondition, which describes the states in which the implementation is allowed to terminate; and a modifies clause, which lists the variables that the implementation is allowed to change. In Figure 1(a), we see a modifies clause that states that method $M$ is allowed to modify $x$ and $y$. Omitted pre- and postconditions default to true and an omitted modifies clause defaults to the empty list of variables. In addition to what's listed in the modifies clause, every routine is implicitly allowed to modify the fields of newly allocated objects, that is, objects allocated since the start of the routine. We treat the object being constructed as being newly allocated in the constructor, and hence the $T$ constructor is allowed to modify $x$ and $y$.

An assert statement gives a condition that is expected to hold whenever execution reaches the statement. A program is erroneous if it can ever reach an assert statement whose condition evaluates to false. In the implementation of method $M$, we've used an assert statement to represent a condition that the programmer may want to rely on (even though the rest of that implementation does not, in fact, rely on the condition). 
A popular view is that an object invariant is simply a shorthand for a postcondition on every constructor and a pre- and postcondition on every public method. The idea behind this view is that an object's invariant should hold whenever the object is publicly visible. This view in itself is appropriate, but is often combined with the following faulty regime:

Callers of $T$ 's methods do not need to be concerned with establishing the implicit precondition associated with the invariant. For the invariant of a class $T$ to hold at entries to its public methods, it is sufficient to restrict modifications of the invariant to methods of $T$ and for each method in $T$ to establish the invariant as a postcondition.

This regime permits a method to violate an object invariant for the duration of the call, as long as it is reestablished before returning to the caller. But, unless every method body is atomic, this is a problem. To illustrate the problem, consider a scenario with a slight variation of $M$ in which the assignments to $x$ and $y$ are separated by a call to some other routine $P$ :

$$
x:=x+3 ; P(\ldots) ; y:=4 * y ;
$$

Assume also that $P$ calls $M$. Note that, at the time $P$ is called, the object invariant for the $T$ object is not certain to hold. Thus, when $P$ calls $M$, the assert statement in $M$ will break. Nothing prevents this reentrancy situation, because the regime lets callers ignore object-invariant induced preconditions, and yet the regime lets implementations rely on the preconditions to hold on entry.

The reasoning is faulty because callers and implementations are not held to the same pre- and postcondition contracts. In particular, the implicit precondition induced by the invariant is not considered by the caller. If instead we make these pre- and postconditions explicit, the mistake becomes apparent. Figure 1(b) shows the previous example program, but with the object-invariant "shorthands" expanded out. In our Larch [18]-like notation, pre- and postconditions are introduced with the keywords requires and ensures, respectively.

Expressing invariants with explicit pre- and postconditions raises a new problem, though: the object invariant of class $T$ is a condition on the internal representation of $T$ objects, the details of which should be of no concern to a client of $T$, the party responsible for establishing the precondition. Making clients responsible for establishing the consistency of the internal representation is a breach of good information hiding practices.

In short, it does not seem prudent either for object invariants to be completely hidden behind information-hiding boundaries or for representation details of object invariants be completely exposed. What we want is for clients to be aware of whether the object invariant holds, without the implementation having to reveal the details of the invariant. We achieve these potentially conflicting goals in our methodology by introducing a publicly available abstraction of whether or not invariants hold.

We note that an analogous problem exists for modifies clauses and modifies checking. We want for clients to be aware of whether a method may change the internal state, with- 
out the implementation having to reveal the details of what the internal state is. We will return to this issue in Section 6.

\section{VALIDITY}

In this section, we introduce one of the basic concepts of our methodology: an explicit representation of when object invariants are known to hold. For now, we ignore subclassing.

For every object, we introduce a special public object field st (for "state") of type $\{$ Invalid, Valid $\}$. If $o . s t=$ Invalid, we say that object $o$ is invalid, and if $o . s t=$ Valid, we say that $o$ is valid. The intent of st is that an object's invariant hold whenever the object is valid. The field is special because it is allowed to appear only in routine specifications, not in invariant declarations or in implementations. An implementation can modify the value of $s t$ only through the use of two new statements, pack and unpack. An object is allocated in the invalid state.

For any class $T$ and object $o$ of type $T$, we define $\operatorname{Inv}_{T}(o)$ as the predicate that holds in a state if and only if the object invariant declared in $T$ holds for $o$ in that state. For example, for the program in Figure 1(a), we have, for any $o$,

$$
\operatorname{Inv}_{T}(o) \equiv 0 \leqslant o . x<o . y
$$

We define the precise meaning of pack and unpack for any expression $o$ of a type $T$ :

$$
\begin{aligned}
& \text { pack } o \equiv \quad \operatorname{assert} o \neq \text { null } \wedge \text { o.st }=\text { Invalid } ; \\
& \text { assert } \operatorname{Inv}_{T}(o) ; \\
& \text { o.st }:=\text { Valid } \\
& \text { unpack } o \equiv \quad \text { assert } o \neq \text { null } \wedge \text { o.st }=\text { Valid } ; \\
& \text { o.st }:=\text { Invalid }
\end{aligned}
$$

The pack statement checks the object invariant and changes st from Invalid to Valid, and the unpack statement changes st from Valid to Invalid.

Since the st field is public, it can be mentioned explicitly in routine specifications, and, in particular, in the preconditions of public methods. Figure 2 shows how the program in Figure 1(a) is written using st. We use this to denote a method or constructor's implicit receiver parameter. The $T$ constructor postcondition says that the constructed object is valid on exit. The method $M$ 's precondition states that clients are expected to call $M$ only on valid objects. Since the modifies clause of $M$ does not include st, it follows that st is still Valid on return from the method.

Because validity is a precondition that applies to all callers, the scenario we considered earlier, where the two assignments in $M$ are separated by a call to a mutually recursive routine $P$, is no longer problematic, because $P$ can call $M$ only when the $T$ object 

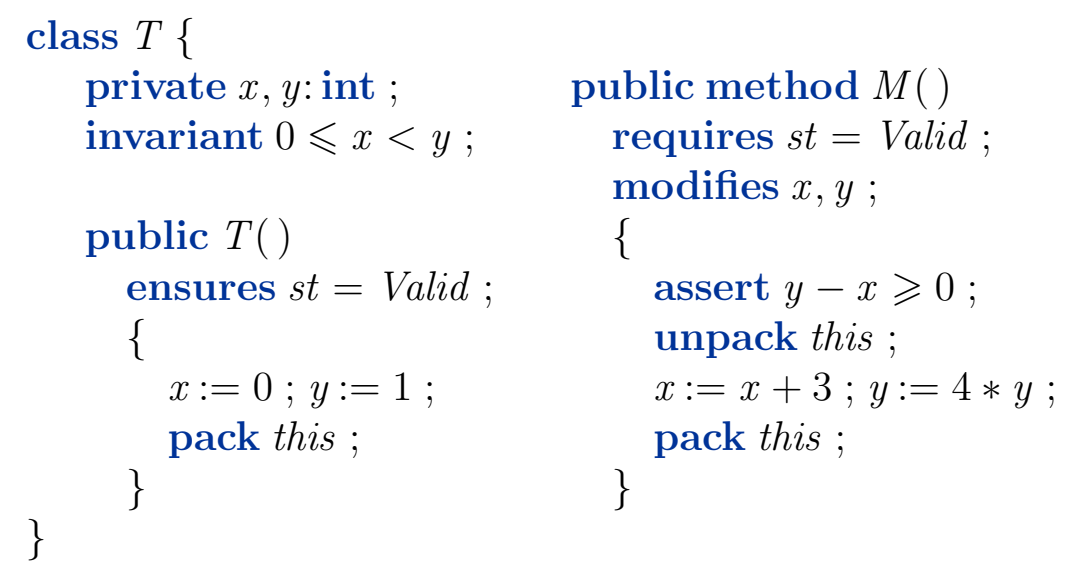

Figure 2: A simple class with an object invariant. Through the use of the special field st, routine specifications detail when the object is expected to be valid. pack and unpack statements are arranged so that fields are updated only for invalid objects.

really is valid. That is, either it is not known in $P$ that the object is valid, in which case the error manifests itself as a failure in $P$ to establish $M$ 's precondition, or $P$ itself requires as a precondition that the object be valid, in which case the error manifests itself as a failure of $M$ to establish $P$ 's precondition.

Note that the asserted condition in $M$, which mentions $x$ and $y$, does not follow literally from $M$ 's precondition, which mentions st. Remember, the intent behind $s t=$ Valid is that the object invariant hold, but we need to impose some restrictions to make sure this is the case.

First, we restrict field updates, because a change to a field can cause the object invariant no longer to hold. The simplest restriction we can think of is to ban field updates for valid objects. Thus, we impose the restriction that a field-update statement $o . f:=E$ is permitted only in states where $o . s t=$ Invalid holds. Only field updates are restricted; our methodology imposes no restrictions on reading fields.

Second, we need to restrict which pieces of the program state an object invariant is allowed to depend on. For now, we restrict each object invariant so that the only part of the state it can depend on are the fields of this (that is, the object whose invariant is being described). Later, we will make this restriction more liberal.

¿From what we now have said, one can prove, by induction over the structure of program statements, that the following is a program invariant, that is, a condition that holds in every reachable program state:

Proposition 0 For the system in this section and any class $T$,

$$
\left(\forall o: T \bullet o . s t=I n v a l i d \vee \operatorname{Inv}_{T}(o)\right)
$$

where o ranges over non-null objects of type $T$. 
Consequently, for method $M$ in Figure 2, we infer from $s t=$ Valid and (1) that the object invariant holds, and thus we know that the asserted condition holds. By the way, it is of no consequence that the assert statement comes before the unpack statement; the fact that the object is valid before the unpack means its object invariant holds there, and since unpack does not change $x$ and $y$, the object invariant also holds just after the unpack.

In summary, we introduced a special field st and two statements pack and unpack to update st, we prescribed new objects to be invalid, we restricted field updates to invalid objects, and we restricted the contents of object invariants. In return, we can rely on program invariant (1) in every reachable program state.

\section{COMPONENTS}

Object-oriented systems are usually built in a hierarchy of layered abstractions, where an object in one layer is implemented in terms of component objects in a lower layer. For example, a buffered input stream may be implemented in terms of a cache and a file reader, the cache may be implemented by an array and some indices, and the file reader may be implemented by a file handle and a set of configuration values. Such layered designs lend themselves to a better separation of concerns and promote reuse. Program correctness may depend not just on relations among fields of one object, but also on relations between the fields of an object and the fields of its component objects. Stating such object invariants is not possible with the restrictions that we imposed in the previous section.

To allow an object invariant to mention indirect access expressions like this.f.g, we need to tackle two problems, which we shall discuss in the context of the following declarations:

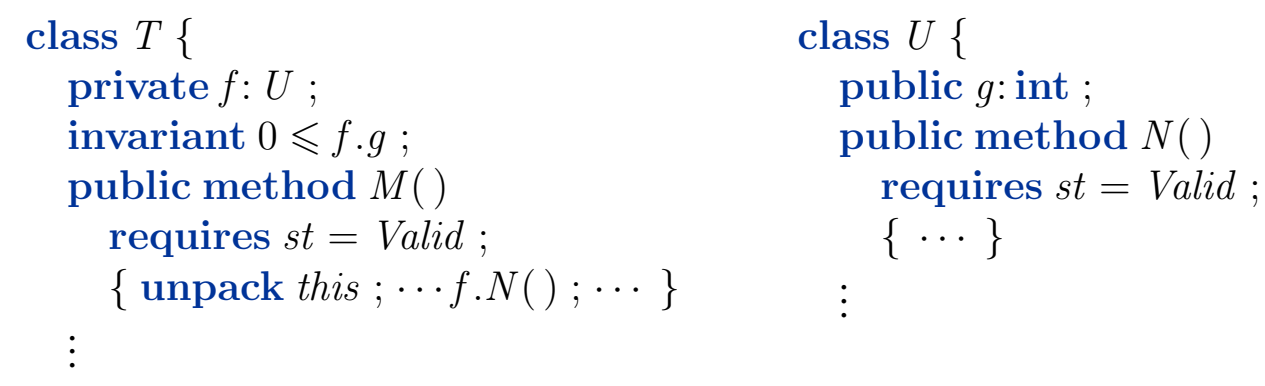

First, consider an update of $u . g$ where $u$ is an invalid object of type $U$. If there is a valid $T$ object $t$ such that $t . f=u$, then the update of $u . g$ may cause $t$ 's invariant to be broken without $t$ being invalid. Therefore, we will arrange for any such $t$ to be invalid whenever $u$ is. To prevent $u$ from being unpacked without regard for the state of $t$, we will make the packing of $t$ put $u$ into a committed state that can be changed back only by unpacking $t$. The committed state indicates that (the object invariant of $u$ holds and that) object $u$ has a unique owning object (in this case, $t$ ). 
Second, consider the call $f . N()$ in method $M$. This call has the precondition $f . s t=$ Valid, which $M$ must somehow establish. One way for $M$ to establish this condition is to add the condition to the precondition of $M$. But if the fact that $T$ uses a field of type $U$ is an internal implementation detail, then mentioning $f$ in the precondition of $M$ would be a breach of good information hiding practices. Note, however, that from what we said in the previous paragraph, we'd like the validity of a $T$ object $t$ to imply the committal of t.f. Hence, we'll arrange for the unpacking of $t$ to take $t . f$ from committed to valid. Now, $M$ can establish the validity of $f$ simply by unpacking this .

Our design decisions in the previous two paragraphs seem appropriate whenever $f$ holds a component of the $T$ object. Then, the committal of $t . f$ to $t$ fits nicely with the idea that $t$ owns $t . f$ and the chains of committed objects form a hierarchy that corresponds to the hierarchy of layered abstractions. If $f$ is not a component of the $T$ object, then the methodology we present in this paper does not allow the fields of $f$ to be mentioned in the class- $T$ object invariant. This is not entirely unreasonable, since the fields of a non-component object may change without regard for the $T$ object whose object invariant would mention them.

To encode the presence of components, we must first provide a way to identify an object's components. For simplicity, we restrict the components to be ones accessible through a field of the object, so we introduce a field modifier rep (for representation [38]) that identifies these fields. Object invariants are now allowed to depend on any field this. $f_{0} . f_{1}, \cdots . g$, where each $f_{i}$ is a field declared with the modifier rep. For example, the object invariant in (2), which depends on this.f.g, is allowed only if $f$ is declared to be a rep field, as in:

private $\operatorname{rep} f: U$;

(Rep fields need not be private.)

Next, we change the type of the special field st to $\{$ Invalid, Valid, Committed $\}$. To redefine the pack and unpack statements, we let $\operatorname{Comp}_{T}(o)$ denote the set of expressions $o . f$ for each rep field $f$ in $T$. Now, for any expression $o$ of type $T$ :

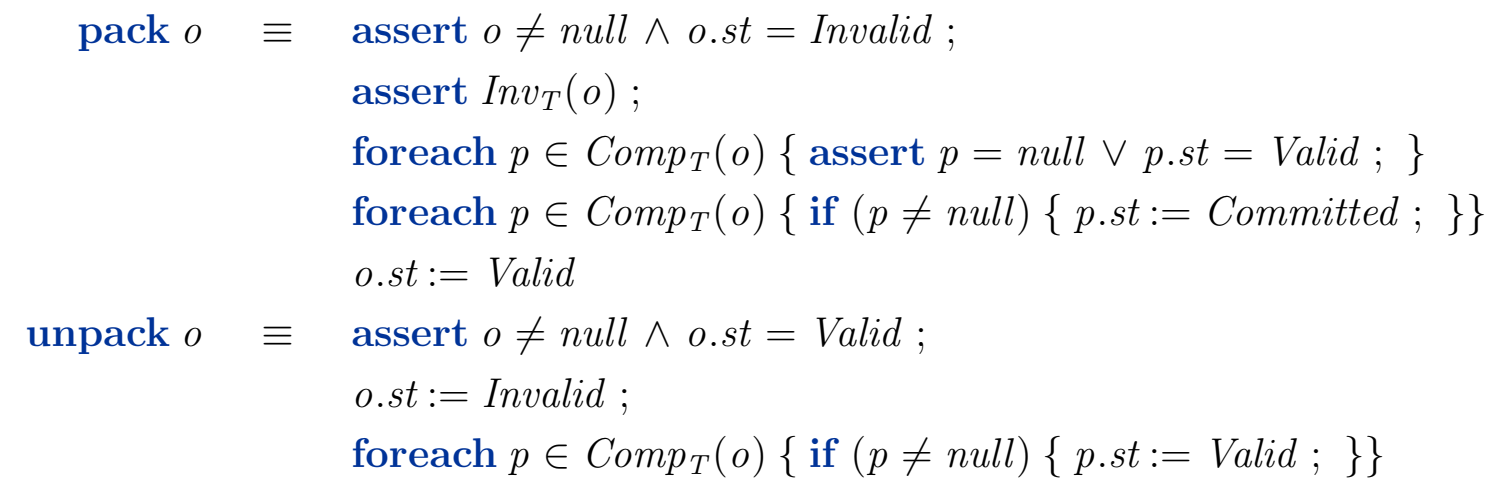

For example, if $T$ is a class with two rep fields, $x$ and $y$, and $o$ is of type $T$, then 
pack $o$ is defined as:

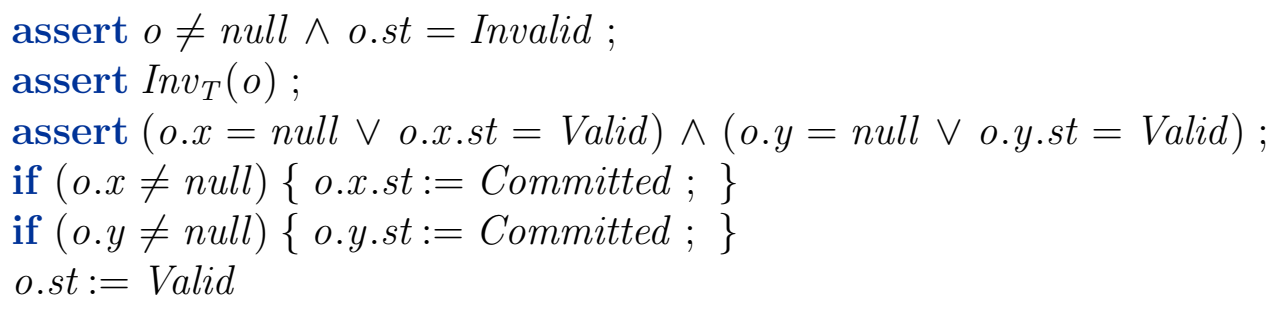

As in the previous section, we prescribe that new objects are allocated in the invalid state and we restrict field updates to invalid objects. One can now prove that the following is a program invariant:

Proposition 1 For the system in this section and any class $T$,

$(\forall o: T \bullet o . s t=$ Invalid $\vee$

$$
\left.\left(\operatorname{Inv}_{T}(o) \wedge\left(\forall p \in \operatorname{Comp}_{T}(o) \bullet p=\text { null } \vee p . s t=\text { Committed }\right)\right)\right)
$$

where o ranges over non-null objects of type $T$.

The proof is by induction over the structure of program statements, where the induction hypothesis also includes a condition that says that committed objects have unique owners:

$$
\begin{aligned}
&\left(\forall o, T, o^{\prime}, T^{\prime}, q \bullet\right.\left(\forall p \in \operatorname{Comp}_{T}(o), p^{\prime} \in \operatorname{Comp}_{T^{\prime}}\left(o^{\prime}\right) \bullet\right. \\
& \operatorname{type}(o)=T \wedge \operatorname{type}\left(o^{\prime}\right)=T^{\prime} \wedge q . s t=\operatorname{Committed}^{\prime} \Rightarrow \\
&\left.\left.p=p^{\prime}=q \Rightarrow o=o^{\prime} \vee o . s t=\text { Invalid } \vee o^{\prime} . s t=\text { Invalid }\right)\right)
\end{aligned}
$$

where $o, o^{\prime}, q$ range over non-null objects, $T, T^{\prime}$ range over types, and type denotes the dynamic type of a given object.

Note, we impose no restrictions on copying object references or on allowing multiple references to a component object. The only restriction is that, at the time of a pack $o$, the components of $o$ (that is, the values of $o$ 's rep fields) are valid, not committed.

Note also that our methodology allows ownership transfer. That is, an object can be owned by different owners over time. For example, if $f$ and $g$ are rep fields of two classes $T$ and $U$, respectively, and $t$ and $u$ are distinct object references of types $T$ and $U$, respectively, then the following code snippet (starting from any state where $t$ and $u$ are both valid) is legal and has the effect of transferring from $t$ to $u$ the component initially stored in $t . f$ :

$$
\begin{aligned}
& \text { unpack } t ; \text { unpack } u ; \\
& u . g:=t . f ; \text { pack } u ; \\
& t . f:=\text { null ; pack } t ;
\end{aligned}
$$

This code snippet also shows a program point, immediately following the packing of $u$, where $u . g$ and $t . f$ both contain the same object reference; this is legal, since $t$ is invalid at that time. 
In summary, we've introduced a field modifier rep that identifies components, we've changed the special field st from the two-valued type it had in the previous section to a three-valued type, we've redefined the pack and unpack statements to update st also for the object's components; we still prescribe new objects to be invalid; and we still restrict field updates to invalid objects. In return, we're able to loosen the restriction on object invariants so that an object's invariant can depend on the fields of its components (and, transitively, the fields of their components). And, we can rely on the program invariant (3) in every reachable program state.

\section{SUBCLASSES}

In this section, we extend our methodology from the previous section to handle subclasses. The idea is that we think of the fields of an object as being divided up into class frames, one for each class from the root of the class hierarchy, object, to the object's dynamic type. For illustration, consider the following class declarations:

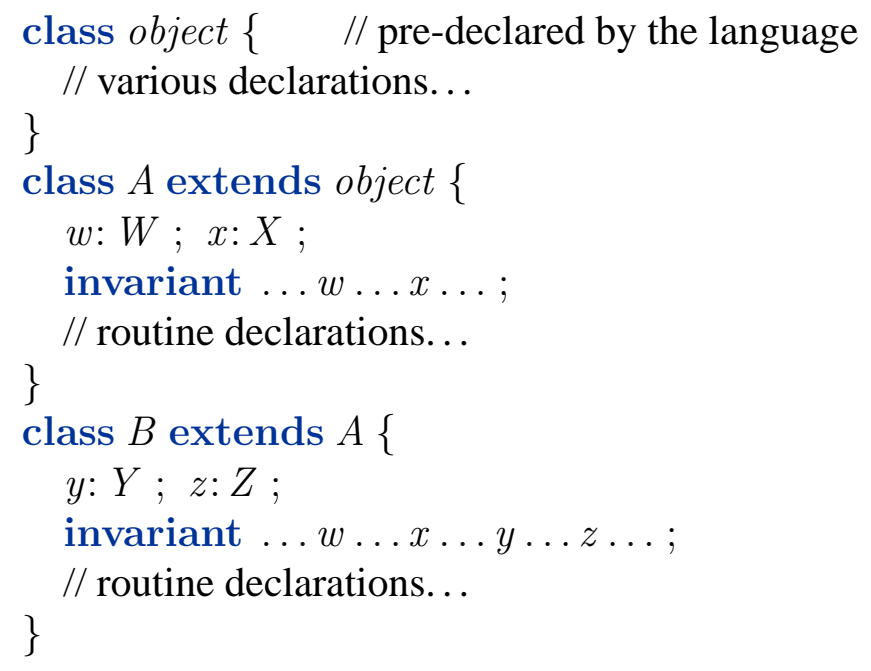

An object of dynamic type $B$ has three class frames: one class frame corresponding to class object, with no programmer-defined fields and the trivial object invariant true; one class frame corresponding to class $A$, with fields $w$ and $x$ and an object invariant that may depend on those fields; and one class frame corresponding to class $B$, with fields $y$ and $z$ and an object invariant that may depend on any of the four fields.

Now, instead of an object being entirely invalid or entirely valid, as in the previous sections, an object can be invalid or valid for each class frame. Therefore, we'd like to keep track of the subset of class frames for which an object is valid. In principle, we could consider any of the $2^{n}$ possible subsets of an object's $n$ class frames, but we will consider only the $n$ subsets that correspond to nonempty prefixes of the sequences of class frames. That is, for an object of dynamic type $B$ in the example classes above, we will let the subset of class frames for which the object is valid be any one of the following subsets: $\{$ object $\},\{$ object, $A\},\{$ object, $A, B\}$. To encode these values, we abandon 
the st field from the previous sections and replace it with two special fields, inv and committed.

To represent the subset of valid class frames, we introduce the special field inv, whose value is the most derived class whose class frame is valid for the object. For example, given the classes above, the inv field of an object of dynamic type $B$ can have one of the values object, $A$, or $B$, representing the three respective subsets shown above.

The special field committed is a boolean that indicates whether the object is committed. We ensure that committed is true only if inv equals the dynamic type of an object.

The division of an object's fields into class frames causes a corresponding division of the pack and unpack statements. For any class $T$ with immediate superclass $S$ and for any expression $o$ whose type is a subclass of $T$, we define:

pack $o$ as $T \equiv$

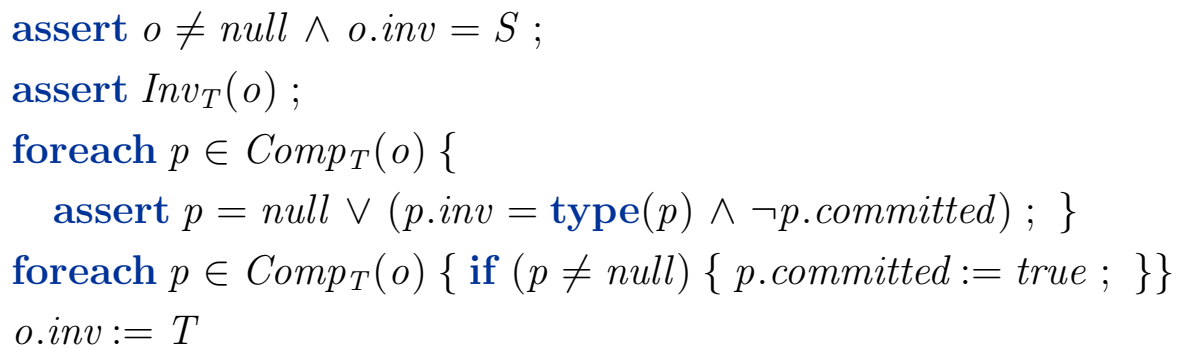

unpack $o$ from $T \equiv$

assert $o \neq$ null $\wedge$ o.inv $=T \wedge \neg$ o.committed;

o.inv $:=S$;

foreach $p \in \operatorname{Comp}_{T}(o)\{$ if $(p \neq n u l l)\{p$. committed $:=$ false ; $\}\}$

In this new encoding, new objects return from the constructor of class object in the state inv $=$ object $\wedge \neg$ committed. Generally, a constructor for a class $T$ typically declares the postcondition inv $=T \wedge \neg$ committed, but our methodology does not insist on this.

The condition under which a field-update statement $o . f:=E$ is permitted is slightly different in the presence of subclasses. If the updated field $f$ is declared in a class $T$, then the statement is permitted only in states where $o$ is "sufficiently unpacked", that is, where o.inv is a strict superclass of $T$.

As before, we allow fields to be declared with the rep modifier. An object invariant in a class $T$ is now allowed to depend on any field of this declared in $T$ or one of its superclasses. For any of those fields that is a rep field, the object invariant is also allowed to depend on any of its fields (and so on, transitively).

¿From what we have said in this section, which is the final version of our methodology, one can prove the following program invariants: 
Theorem 2 For the system in this section, the following conditions are program invariants:

$$
\begin{aligned}
& (\forall o, T \bullet o . c o m m i t t e d \Rightarrow o . i n v=\operatorname{type}(o)) \\
& \left(\forall o, T \bullet o . i n v<: T \Rightarrow \operatorname{Inv}_{T}(o)\right) \\
& \left(\forall o, T \bullet o . i n v<: T \Rightarrow\left(\forall p \in \operatorname{Comp}_{T}(o) \bullet p=\text { null } \vee p . c o m m i t t e d\right)\right) \\
& \left(\forall o, T, o^{\prime}, T^{\prime}, q \bullet\left(\forall p \in \operatorname{Comp}_{T}(o), p^{\prime} \in \operatorname{Comp} T_{T^{\prime}}\left(o^{\prime}\right) \bullet\right.\right. \\
& \left.\left.\quad \text { o.inv }<: T \wedge o^{\prime} . i n v<: T^{\prime} \wedge q . \operatorname{committed} \wedge p=p^{\prime}=q \Rightarrow o=o^{\prime} \wedge T=T^{\prime}\right)\right)
\end{aligned}
$$

where quantifications over references ranges over non-null objects, and where we're using $<:$ to denote the reflexive and transitive subclass relation.

Proof sketch. We sketch the proof here; the full proof is given in Appendix A. The proof shows that the conditions are maintained by each of the four program statements that can extend the ranges of the quantifications (by allocating objects) or change the values of object fields: (a) the object constructor, (b) the pack statement, (c) the unpack statement, and (d) field update. The proof relies on the fact that static type checking guarantees that an object-valued expression of type $T$ yields either a null reference or a reference to an object whose dynamic type is a subclass of $T$. Most of the cases are simple; three cases are slightly more involved. First, the proof of (5) for case (d) uses the fact that invariants can only mention access expressions of the form this. $f_{0} . f_{1} \cdots . f_{n}$ where the $f_{i}(i<n)$ are rep fields, and then uses $n$ applications of (4) and (6). Second, the proof of (6) for case (c) uses (7). Third, the proof of (7) for case (b) uses (6). (End of Proof sketch.)

In summary, our methodology

- introduces a field modifier rep that identifies components,

- restricts the contents of an object invariant in a class $T$ to depend only on the object's fields in $T$ and its superclasses, and (transitively) on the fields of rep fields,

- keeps track of the class frames for which an object's invariant is valid and whether the object is committed,

- provides the statements pack and unpack for changing the validity and committal states of objects,

- prescribes new objects to return from the object constructor as uncommitted and with only the object class frame as valid,

- permits field-update statements only for sufficiently unpacked objects, and

- guarantees, therefore, that program invariants (4)-(7) hold in every reachable program state. 


\section{ROUTINE SPECIFICATIONS}

A routine's specification is a contract between its callers and its implementations. It describes what is expected of the caller at the time of the call and what is expected of the implementation at the time of the return. Describing such contracts is not always easy. In this section, we describe two innovations in writing and interpreting routine specifications. The first innovation uses the notion of committed objects to provide a solution to the problem of specifying what state a routine may modify. The second innovation provides a way for the specification of a dynamically dispatched method to talk about the entirevalidity of an object without forcing implementations to reason about possible subclasses.

\section{Writing modifies clauses}

To be useful to a caller, a routine specification has to determine which pieces of the program state may be modified by the routine and which may not. This is tricky, because most variables are not accessible in the declaration of a routine. For example, the variables in scope at an eventual caller may not be known to, let alone nameable by, the scope that gives the routine specification. Conversely, the variables used by a routine's implementation may be private implementation details which, in the interest of good information hiding, are not to be mentioned explicitly in a public routine specification. In fact, in a layered program design, the implementation details are contained in several layers of abstractions and may not even be nameable by the routine.

To accomplish our goal of specifying modifications, we model the heap of the objectoriented program by a global variable Heap, whose type is a two-dimensional array of values, indexed by object identities (references) and field names [39]. For example, a program's access expression o.f denotes a particular location in the heap. We model this location in the heap by the expression Heap $[o, f]$. Consequently, we can quantify over field names. For example, we can rewrite the quantification over $p$ in (6) as:

$$
(\forall f \in \operatorname{RepFields}(T) \bullet \operatorname{Heap}[o, f]=\text { null } \vee \operatorname{Heap}[\operatorname{Heap}[o, f], \text { committed }])
$$

where RepFields $(T)$ is the set of names of rep fields in $T$.

As we've seen above, we use modifies clauses to specify modifications. The modifies clause gives a list of access expressions that, evaluated in the routine's pre-state, gives a set of heap locations that the routine is allowed to modify (or, more precisely, to have a net effect on-a routine is allowed to modify any heap location temporarily if it restores the original value before returning). It is common also to allow the routine to allocate new objects and modify their state ( $c f$. [25]). If we use a special boolean field alloc to denote which objects have been allocated, then a modifies clause $W$, according to what we've said so far, is interpreted as the following postcondition:

$$
(\forall o, f \bullet H e a p[o, f]=\operatorname{old}(H e a p[o, f]) \vee(o, f) \in \operatorname{old}(W) \vee \neg \operatorname{old}(\text { Heap }[o, \text { alloc }]))
$$

This says that either the contents of heap location $(o, f)$ is unchanged, or $(o, f)$ is a heap location explicitly mentioned in the modifies clause, or $o$ is an object that was not 
allocated on entry to the routine. But even this does not account for the modification of layers of private state.

Our innovation is to allow every routine also to modify the fields of committed objects. This policy lets the routine modify layers of private state without explicitly mentioning this state in the modifies clause. We formalize our policy as the following postcondition, for any modifies clause $W$ :

$$
\begin{aligned}
(\forall o, f \bullet & H e a p[o, f]=\operatorname{old}(H e a p[o, f]) \vee(o, f) \in \operatorname{old}(W) \vee \\
& \neg \operatorname{old}(\text { Heap }[o, \text { alloc }]) \vee \operatorname{old}(\text { Heap }[o, \text { committed }]))
\end{aligned}
$$

Note, our policy lets a routine modify not just layers of private state, but also the state of any object that happens to be committed at the time the routine is called, which may seem quite liberal. The intuition for why this policy is in fact not too liberal is that when an object is committed, one should rely only on what object invariants say about the object's state, not on any other details of its state. Of course, in order to actually modify a field of an object, the object must be unpacked. Therefore, a routine that seeks to modify the fields of a committed object $o$ must first arrange for each of $o$ 's transitive owners to be unpacked and then, once $o$ 's owner is unpacked and $o$ is decommitted, unpack $o$ itself. Moreover, unless such a routine is allowed to have a net effect on the inv and committed fields that are modified during this unpacking process, the routine must see to it that these objects are repacked, which will cause our methodology to enforce that the invariants of these objects hold.

As a final extension, we allow a modifies clause to mention special expressions of the form $E .\{T\}$, where $T$ is either a class name or an expression of the form type $(o)$ for an object-valued expression $O$. Such an expression $E .\{T\}$ denotes all programmerdefined fields of object $E$ declared in class $T$ and its superclasses.

\section{Writing preconditions of methods and overrides}

Our methodology divides an object's state into class frames, which allows object invariants to be stated and maintained independently for each class. This flexibility comes at a price, namely that each unpack statement needs to state which type it is unpacking from. This obligation is easily met by giving an appropriate precondition of the routine that performs the unpack. For example, to make sure a routine implementation can execute the statement unpack this from $T$, the routine can declare the precondition $i n v=T$. But having to state in a precondition the exact value desired for an object's inv field seems incompatible with dynamically dispatched methods.

Our innovation is to introduce a special expression, 1 , for use in the specification of dynamically dispatched methods. The idea is that for the caller of the dynamically dispatched method, 1 means type(this), and for an implementation of the method given in class $T, 1$ means $T$. Thus, if a method has the precondition $i n v=1$, a caller invokes the method on an object that is entirely valid, without having to know the dynamic type of the object. This entirely-valid state is, for example, the state a component object 
is in immediately after the owner has been unpacked. And an implementation of this method in a class $T$ assumes on entry that $i n v=T$, which allows it to perform an unpack this from $T$ statement.

For reasoning through routine specifications to be sound, routine specifications must be interpreted the same way for callers as for implementations. Ostensibly, our idea about 1 may therefore seem untenable for sound reasoning. Let us be more precise about our scheme for treating dynamically dispatched methods:

The declaration of a dynamically dispatched method $M$ introduces a procedure named $M$, whose specification is that of the method but with 1 replaced by type(this). The implementation of $M$ in a class $T$ introduces a procedure named $M @ T$, whose specification is that of the method but with 1 replaced by $T$. The body of procedure $M$ is supplied by the run-time system; it looks at the dynamic type of this, call it $T$, and then calls the corresponding procedure $M @ T$. Note that the precondition of this procedure $M @ T$ follows from the precondition of procedure $M$, since type $($ this $)=T$. The body of procedure $M @ T$ is the implementation of $M$ given in $T$, modulo superclass calls; if $T$ has immediate superclass $S$, then any call super. $M(\ldots)$ to the superclass implementation of $M$ is replaced by a call to procedure $M @ S$. Any invocation of the dynamically dispatched method is replaced by a call to procedure $M$.

This scheme relies on the existence of certain procedures $M @ T$ and $M @ S$, so we impose a restriction on programs that every class supply an implementation for every dynamically dispatched method declared in the class or in a superclass. Such implementations can, of course, consist just of a call super. $M(.$.$) or of the code sequence$

unpack this from $T$; super. $M(.$.$) ; pack this as T$;

In this scheme for treating dynamically dispatched methods, which is really quite close to actual implementations of such methods, note that the specification for each procedure is interpreted the same way for its callers and its body, and hence we achieve sound reasoning.

Finally, having introduced 1 in the specification of dynamically dispatched methods to stand for either type(this) or a particular class name $T$, we also allow expressions of the form $E .\{1\}$ in modifies clauses. In the next section, we show our specifications in use.

\section{EXAMPLES}

\section{Readers}

Figure 3 shows a reader, an input stream that produces characters. Different subclasses of Reader draw their characters from different sources; for example, a file reader draws its characters from a file in the file system, and an array reader draws its characters from a character array in memory (cf. [28]). The GetChar method returns a reader's characters, encoded as integers. When the reader's supply of characters has been exhausted, 


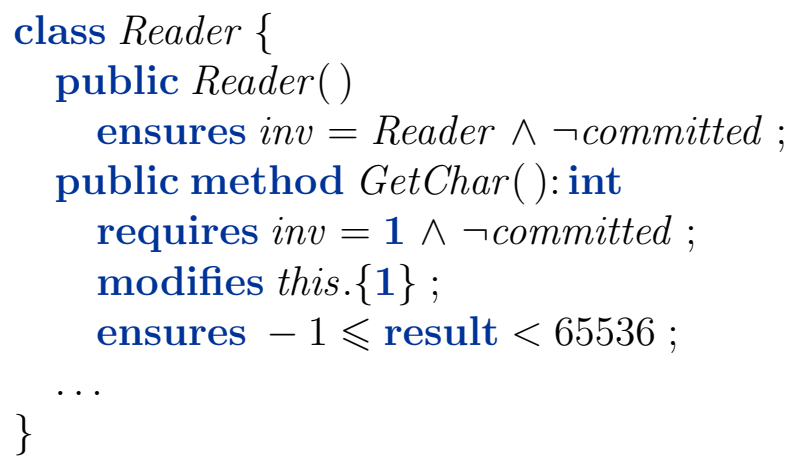

Figure 3: The example class Reader, whose GetChar method produces characters.

GetChar returns -1. The Reader class in Figure 3 shows the typical specifications in our methodology of a constructor and a dynamically-dispatched method (except the postcondition of GetChar, which is specific to that method).

The constructor postcondition says that the constructed object will be valid for class Reader. Thus, if the caller is the constructor of a further subclass $R$, then that constructor gets to know enough of the state of the object to perform a pack this as $R$ statement. If the caller is a client that invokes new Reader ( ), then that client gets to know enough of the state of the object to invoke a method that requires inv to equal the dynamic type of the object. This state is also what is required in order to pack an object that uses the reader as a component.

The method precondition allows the implementation of the method in any subclass $T$ to unpack the object from $T$. After the unpack operation, the fields of the object declared in $T$ can be modified and the method's implementation in the superclass can be called. The modifies clause this. $\{\mathbf{1}\}$ allows the method implementation in a class $T$ to modify all programmer-defined fields on the object declared in superclasses of $T$. For example, when a method implementation invokes the implementation of the method declared in the direct superclass, then this modifies clause ensures that the fields of the calling class are not affected by the superclass call. Since the special fields inv and committed are not programmer defined, the modifies clause this. $\{1\}$ implies that as much can be said about the reader's invariants after the call as can be said about them before the call.

\section{Array readers}

Figure 4 shows the declaration of a Reader subclass. An array reader is initialized with an array of characters, and its GetChar method returns these characters, one by one. The object invariant's constraint on $n$ guarantees, together with the precondition about inv, the absence of array bounds errors in the implementation.

An important decision in the design of the constructor is whether or not the constructor may capture the given array, or if it has to make a copy of the array (cf. [13]). Our 


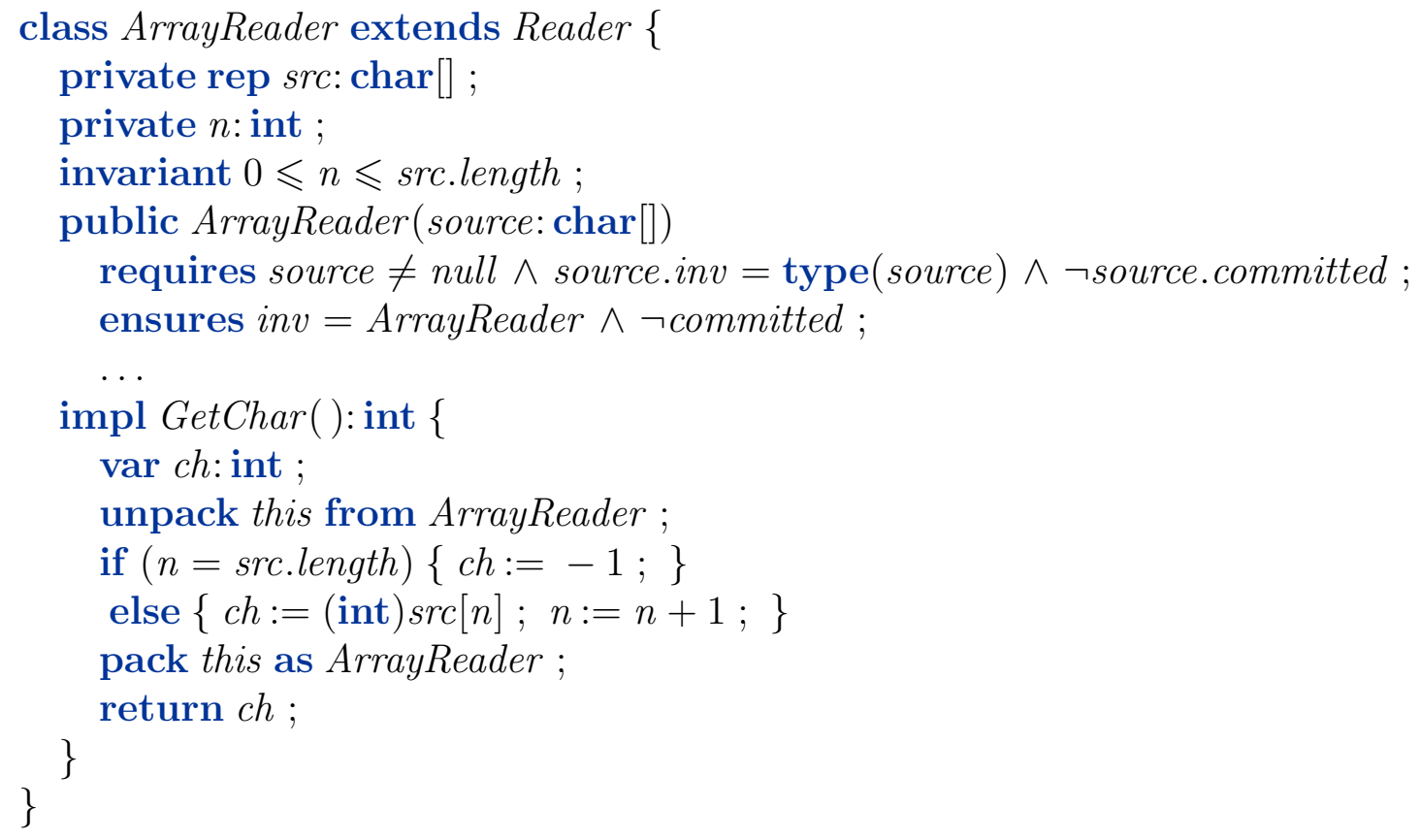

Figure 4: A Reader subclass that produces its characters from a given array.

methodology makes this decision explicit. The specification in Figure 4 effectively forces the constructor implementation to make a copy of the array. To see why that is, consider the following attempted implementation, which captures the array:

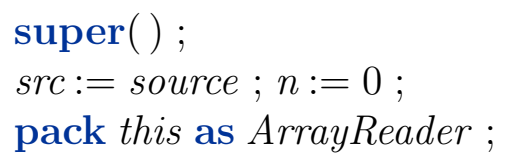

This code does not meet the constructor's specification, because it modifies the special field source.committed (from false to true), which is not allowed by the given (empty) modifies clause. This implementation would be allowed, however, if the specification were changed also to include

\section{modifies source.committed ;}

In that case, the caller is alerted that it cannot retain ownership of the array. But note that the caller may retain a reference to the array (in contrast to linear type systems and to alias burying [6], for example), as long as the reference is not used as an owned reference.

\section{Lexers}

In the lexer-reader example [13], a lexer produces a stream of tokens from a stream of characters, see Figure 5. 


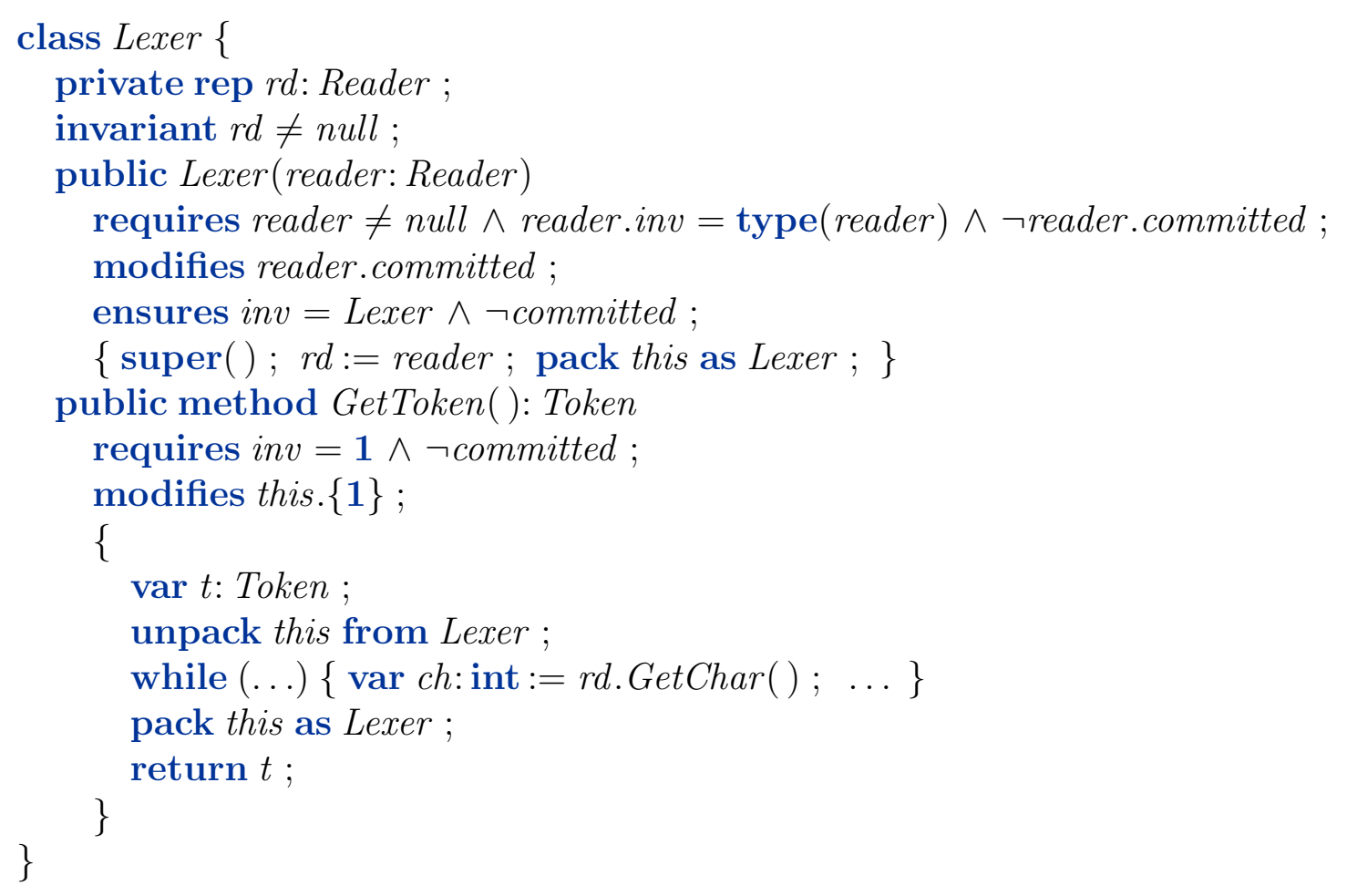

Figure 5: A lexer consumes characters and produces tokens.

The constructor is specified to allow its implementation to capture the given reader. Unlike Detlefs et al. [13], our methodology allows the field $r d$ to be private; it need not be exposed in the public interface. Our methodology allows a lexer client to retain a reference to the reader it passes to the Lexer constructor, but this is harmless: when this reader is committed, the caller cannot rely on the reader's fields being unchanged across routine invocations.

GetToken's modifies clause allows the fields of the lexer to be modified, but it does not explicitly list the modifications it causes on the fields of the underlying reader. These modifications are still allowed, however, since $r d$ is committed on entry to GetToken .

Finally, we point out that the Lexer class can include a method that relinquishes the underlying reader. To be useful to a client, such a method must relinquish the reader in an uncommitted state, which effectively means that the lexer needs to give up ownership of the reader. In the following possible method declaration, the lexer is left in an inconsistent state after relinquishing the reader, putting the lexer in a state where GetToken cannot be invoked. Note that it is not necessary for the implementation to set $r d$ to null (although 


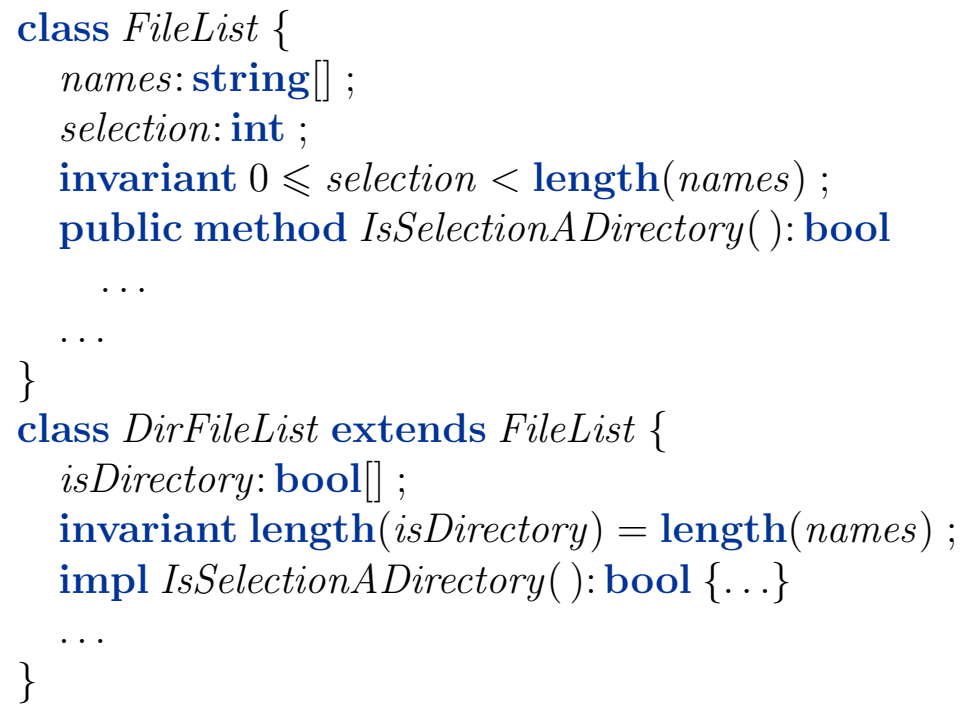

Figure 6: A class FileList and its subclass DirFileList, whose invariant depends on a field in the superclass.

doing so may be a good idea to achieve good garbage-collector performance).

public method RelinquishReader ( ): Reader

requires inv $=1 \wedge \neg$ committed ;

modifies this. $\{\mathbf{1}\}$, inv ;

ensures result $\neq$ null $\wedge$ result.in $v=$ type $($ result $) \wedge \neg$ result.committed ;

\{ unpack this from Lexer; return $r d ;$ \}

\section{Final methods}

As a final example, we consider a tricky, and perhaps understudied, issue related to methods that are not dynamically dispatched, so-called final methods. We discuss how final methods can be supported in our methodology.

Consider the two classes in Figure 6. Objects of class FileList have a nonempty list of files in a file system and an index into that list to denote a currently selected file. The class also provides a method for determining whether or not the current selection is a directory. The subclass, DirFileList, has an additional list that keeps track of which of the files are directories in order to provide a more efficient implementation. Note that the invariant in the subclass depends on a field declared in the subclass (isDirectory) and a field declared in the superclass ( names).

Consider the following FileList method, which the designer has designated as final:

public final method ResetSelection() requires $i n v=$ FileList $\wedge \neg$ committed ;

$\{$ unpack this from FileList ; selection $:=0$; pack this as FileList ; \} 
While this code will verify, the precondition $i n v=$ FileList does not make the method particularly useful to callers. A typical situation would be that the caller has a variable $x$ of type FileList and knows $x . i n v=\operatorname{type}(x)$. However, if the dynamic type of $x$ is a strict subclass of FileList, like DirFileList, then the caller would have to do some unknown amount of unpacking before invoking the ResetSelection method.

Alternatively, by instead using the precondition inv = type (this), the burden of unpacking will rest with the method implementation. Still, the number of required unpack operations to change inv from type(this) to FileList is unknown. To make this situation easier, we may consider two new statements pack* and unpack ${ }^{*}$, to be used as follows:

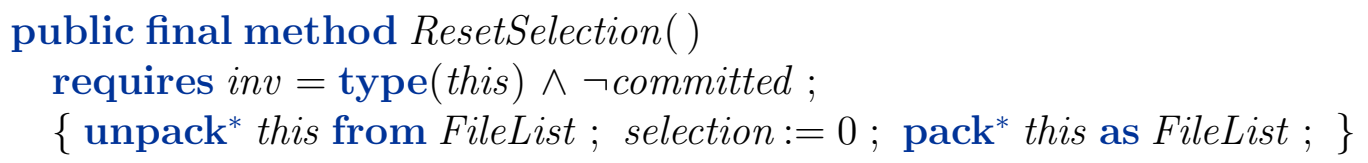

The idea here is that the unpack* statement unpacks the object from an entirely-valid state $($ inv $=$ type $($ this $)$ ) to the immediate superclass of FileList , and the pack* statement does the reverse, packing the object back to a entirely-valid state.

While the unpack* statement is unproblematic, the pack* statement needs to check the invariants declared in all classes from FileList to the dynamic type of the object. Checking those invariants requires having information about what the invariants are, which poses a problem in modular verification where the possible dynamic types of the object are not known.

By restricting what such invariants can depend on, however, it is not necessary to know the exact details of the invariants. In the example, we only need to know that the invariants of strict subclasses do not depend on the selection field. That is, if we restrict the fields that can be modified after an unpack* statement, the pack* statement still just needs to check the invariant in the given class (here, FileList). For example, private fields of the given class can always be modified without the risk of breaking the invariants of strict subclasses, since those subclasses cannot access the private fields, and therefore cannot mention these private fields in their invariants. One can also allow non-private fields to be modified, as long as these fields are marked with a special declaration that says they cannot be depended on by invariants of strict subclasses ( $c f$. [31]).

The problem we have illustrated in this subsection may at first seem just a technical difficulty in our methodology. Upon reflection, however, it seems to be bringing out an important issue in the design of object-oriented software: final methods do not give subclasses a chance to augment the method's behavior to accommodate subclass invariants, and therefore it is prudent for a final method to modify only those fields a subclass is known not to depend on, unless the modifications occur via invocations of dynamicallydispatched methods. In existing programming languages, even Eiffel which includes invariants, there are no such restrictions on what final methods can modify; application of our methodology points out this problem.

In general, our inv field is used to specify three kinds of conditions, in preconditions for example. First, the precondition $i n v=1$ is appropriate for dynamically-dispatched 
methods that either do the work themselves or call superclass implementations of the method to do the work (in the tool Fugue [11, 12], methods with this kind of behavior are called sliding methods). Doing the work, of course, means being able to unpack in order to update fields. Second, the precondition $i n v=$ type $($ this $)$ is appropriate for methods whose implementations do not unpack the object, but instead call other methods of either the first or second kind. Third, the precondition $i n v=T$ for a particular class $T$ is appropriate for private or protected (that is, used by subclasses) subroutines in class $T$. These methods may do some work themselves and may call such subroutines in superclasses, but cannot invoke dynamically-dispatched methods of the first or second kind. The modifies clauses for these three kinds of methods are often this. $\{1\}$, this. $\{$ type $($ this $)\}$, and this. $\{T\}$, respectively. Having the programming language provide labels for the methods according to which of these three kinds of uses the programmer intends may seem a good idea, even when the actual verification of object invariants is not of interest.

\section{RELATED WORK}

Our methodology draws from two main lines of research. First, it draws from the methodology developed for the Extended Static Checker for Modula-3 (ESC/Modula-3) [14]. The ESC/Modula-3 methodology encodes object invariants idiomatically: the invariant condition is represented by a programmer-declared boolean abstract field valid whose meaning is defined as a function of the object's state by a representation declaration [28]. Routine specifications then mention an object's valid field explicitly. The main difference with our methodology is that the abstract field valid changes implicitly, in sync with any update of the state that makes up the representation of valid. In fact, when a program updates a field o.f, then the abstract valid fields of all transitive owners of $o$ might change. In contrast, our methodology uses an auxiliary variable inv that permits us to distinguish object states where a particular invariant must hold from object states where the invariant is allowed to be violated. Because we allow field updates only for unpacked objects and because we arrange for owning objects to be unpacked whenever their owned objects are unpacked, a change in a field can occur only at times when all possibly affected object invariants are allowed to be violated. As another difference, our methodology more easily allows a subclass to extend the invariant declared in a superclass. A similar comparison can be made between our methodology and Müller's methodology [35], which draws some inspiration from the ESC/Modula-3 methodology and improves it, for example by providing a proof of soundness.

The second line of research that our methodology draws from is the work on Vault [10] and Fugue [11] building on research from Alias Types [40] and separation logic [23]. The components in our methodology correspond closely to existentially bound store fragments in these systems. Pack and unpack operations correspond to existential introduction and elimination. All these approaches make state invariants explicit in pre- and postconditions. Typestates in Fugue [12] extend such invariants to handle subclasses and include the notion of sliding methods. Invariants in Fugue are simpler though and cannot span 
multiple class frames. Modifies clauses in Fugue contain the implicit permissions granted to components, but are generally less expressive. One advantage of the methodology presented in this article is that it requires no special separation logic. As a result, it is currently more amenable to mechanical theorem provers based on first-order logic.

The desire to avoid representation exposure and unwanted aliasing has led to a line of research on systems to control aliasing (e.g., [21, 0, 34, 9, 4, 6, 15, 8]). Our uniquely owned components can be seen as a variation on these systems. Many of these systems control aliasing through a static type system, which makes ownership transfer harder to achieve. A more important difference is that these other systems are not combined with a methodology to guarantee properties about detailed object invariants.

Universe types and the associated methodology for proving object invariants in Müller's thesis $[36,35]$ also prevent problems with reentrancy, but does so through more drastic restrictions than our methodology. In Müller's system, reentrancy is allowed only through read-only pointers without any object-invariant guarantees (see also [37]).

Our formulation of modifies clauses takes advantage of the component structure needed for invariants. If one needs more flexibility in specifying which heap locations may be modified by a routine, one can use something like explicit data groups $[26,30]$ in conjunction with our approach. Other work has also considered various effect systems for object-oriented programs (e.g., [41, 17]).

Huizing and Kuiper consider a proof system for object-oriented programs with object invariants [22]. Rather than relying on ownership to confine which access expressions may be mentioned in invariants, their system uses the declared invariants of other classes to determine if a method may violate those invariants. This limits what can be verified modularly. Moreover, in their system, all invariants of all objects must hold on all method boundaries, which we consider to be too strong of a restriction.

The Java Modeling Language (JML) [24] is a specification language for Java programs and includes object invariants. JML combines the design by contract approach of Eiffel [33] with the model-based specification approach of Larch [18]. Like the Eiffel compiler, the JML compiler turns JML specifications into run-time checks. Another tool in the JML family [7] is the Extended Static Checker for Java (ESC/Java) [16], which checks JML specifications statically. ESC/Java uses heuristics to determine which object invariants to check at method invocations. Described in detail in the ESC/Java user's manual [29], these heuristics are a compromise between flexibility and likelihood of errors and do not guarantee soundness.

Banerjee and Naumann [1] consider what it means, formally, for the exported interface of a class to be independent of the implementation of the class, which may rely on object invariants. Their semantic results are sound even in the presence of call-backs, but just how one goes about establishing the antecedents of their theorems is mostly left unaddressed.

The object-based language CLU [32] uses types to distinguish the external view of an object from its internal representation view. A program can convert between the two views using operations that correspond to our pack and unpack statements. The CLU 
methodology is not formalized and leaves the absence of certain errors (like rep exposure, which refers to an unwanted sharing of the underlying data structure) as a responsibility of the programmer. Wills outlines an object-oriented programming system where a type's invariant always holds [41]. His formalization allows the distinction between such a type and the possibility that an object's invariants are violated during the course of a method. His thesis identifies problems with aliasing, reentrancy, and modifies clauses, but leaves full solutions to future work.

In a previous version of this work, we presented a slightly more general treatment of components [2]; the current presentation using rep was chosen for simplicity. Leino and Müller have extended our methodology to allow invariants for more kinds of data structures, including cyclic data structures [27]. Barnett and Naumann provide another extension that lends itself to specifying and verifying even more programs, including a common subject-view pattern [3]. They make use of an update guard, which allows a programmable level of abstraction over the conditions on which an object invariant can depend.

Our use of unpack and pack operations with object invariants is in several ways similar to the use of acquire and release operations with monitor invariants in a concurrent program [20]. The monitor invariant is known to hold when no thread is executing within the monitor, that is, between acquire and release operations. Provided the monitors are not thread-reentrant (unlike those in Java and .NET), the monitor invariant is thus known to hold on entry to a monitor and is checked to hold on exit from the monitor. It would be interesting to investigate the adaptation of our methodology to concurrent programming, since, for example, our methodology provides support for reasoning about object references and reentrancy (see, e.g., [5] for another methodology for concurrent programming based on ownership).

\section{CONCLUSIONS}

Our conclusion is that reentrancy in object-oriented programming makes it untenable to treat object invariants as implicit pre- and postconditions that completely hide the preconditions from callers. In order to be useful, it must always be clear to a programmer at what program points an object invariant can be relied upon. By enriching the program's state space with auxiliary variables that say which object invariants can be relied on and which object invariants are allowed to be violated, our methodology provides programmers with a flexible and precise way to specify their intentions about object invariants.

Though our methodology organizes objects into a hierarchy of owned components, it is perhaps surprising how liberal it is about confining object aliases. An object's components do not have to be protected from being aliased; references to them can be freely copied. Our only restriction is that each object have at most one valid owner at any one time, which is enforced at the time of pack operations. Moreover, while our methodology restricts field updates, there are no restrictions on reading the fields of any object. However, without knowledge about the state of the object's invariants, the values read from an 
object's fields may be meaningless when reasoning about the program's correctness.

As we have presented it, our methodology makes programs look rather verbose, with many pack and unpack statements and with routine specifications that mention inv and committed. We'd like to explore syntactic sugar and useful defaults for making programs more concise. Such defaults may for instance take advantage of the fact that public dynamically-dispatched methods tend to have a precondition of inv $=\operatorname{type}($ this $) \wedge$ $\neg$ committed and that their implementations tend to consist of some block of code bracketed by unpack and pack statements. In the meantime, the explicit primitives in this paper accommodate further exploration of variations and extensions of our methodology.

In short, we have provided a modular verification methodology for object-oriented programs that is both sound and sufficiently expressive to deal with object invariants without sacrificing proper data encapsulation. We are currently implementing a checker that supports this methodology, and we look forward to putting the methodology to the test when checking invariants on existing Microsoft code.

Acknowledgements We thank the participants at the Formal Techniques for Java-like Programs workshop (FTfJP 2003) for comments and discussion. Thanks also to Ernie Cohen whose listening ears on a bus helped improve the presentation of this material. Dave Naumann, Sriram Rajamani, and the referees all provided useful comments on previous versions of this paper.

\section{A SOUNDNESS}

In this appendix, we give the full proof of the soundness theorem.

Proof of Theorem 2. The proof shows that the conditions (4)-(7) are maintained by each of the four program statements that can extend the ranges of the quantifications (by allocating objects) or change the values of object fields: (a) the object constructor, (b) the pack statement, (c) the unpack statement, and (d) field update.

Case (a): the object constructor. The object constructor sets o.committed to false for the newly allocated object $o$, hence establishing (4). It sets o.inv to object and thus establishes (5), since $\operatorname{Inv}_{\text {object }}(o)$ is just true (that is, class object has no object invariant). Similarly, since class object has no rep fields (that is, $\operatorname{Comp}_{\text {object }}(o)$ is the empty set), the constructor also establishes (6) and (7).

Case (b): the pack statement. For each p.committed that the pack statement sets to true, there is a corresponding precondition that $p$ is entirely valid. Moreover, the precondition $o . i n v=S$, the condition type $(o)<: T$ (which follows from the fact that static type checking ensures that the dynamic type type $(o)$ is a subclass of the static type of $o$, and checks that the static type of $o$ is a subclass of $T$ ), and the invariant (4) in the pre-state together imply $\neg$ o.committed. Hence, the pack statement maintains program invariant (4). 
The statement's precondition $\operatorname{Inv}_{T}(o)$ guarantees that changing o.inv to $T$ from the immediate superclass $S$ maintains (5). The statement sets p.committed to true for every non-null component $p$ in class $T$, so (6) is also maintained.

To prove the maintenance of (7), consider an instantiation of $o, T, o^{\prime}, T^{\prime} q, p, p^{\prime}$. We consider two cases. First, suppose q.committed is false in the pre-state of the pack statement. Then, by (6), there's no $o, T, p$ such that

$$
\text { o.inv <: } T \wedge p \in \operatorname{Comp}_{T}(o) \wedge p=q
$$

holds in the pre-state. Therefore, if the antecedent of (7) holds in the post-state, then $O$ and $o^{\prime}$ both refer to the object given in the pack statement (which is the only object whose inv field is changed), and thus the consequent holds in the post-state. Second, suppose q.committed is true in the pre-state. Then, from the antecedent of (7) and the precondition of the pack statement, we conclude that neither $o$ nor $o^{\prime}$ refers to the object on which the pack statement is invoked. Thus, the antecedent does not change and (7) is maintained.

Case (c): the unpack statement. Program invariant (4) is maintained, because the unpack statement changes committed only from true to false and it changes inv only for an uncommitted object. Program invariants (5) and (7) are maintained, because the statement only has a weakening effect on these predicates.

For the maintenance of (6), note that the statement changes p.committed (from true to false) only when $p \in \operatorname{Comp}_{T}(o)$, where $o$ and $T$ are the arguments to the unpack statement. Since for this $o$ and $T$, the statement changes o.inv to falsify the antecedent of (6), it only remains to be proved that there is no other $o^{\prime}, T^{\prime}, p^{\prime}$ satisfying $p^{\prime} \in \operatorname{Comp}_{T^{\prime}}\left(o^{\prime}\right)$ such that $p^{\prime}$.committed is changed by the statement. By program invariant (7) in the pre-state, we have $o=o^{\prime} \wedge T=T^{\prime}$ for any such $o^{\prime}, T^{\prime}, p^{\prime}$, which concludes the proof.

Case (d): field update. The field update statement cannot be used to change the special fields inv and committed, so it maintains (4).

Let $f$ be a field declared in a class $F$, and consider a field update statement $x . f:=E$. Suppose the statement has an effect on $\operatorname{Inv}_{T}(o)$ for some $o$ and $T$. That means the object invariant declared in class $T$ mentions a access expression that denotes $x$. $f$. We consider any such access expression going through non-null objects. Such an access expression in the object invariant has the form this. $g_{0} . \cdots . g_{n-1} . f$ for some rep fields $g_{0}, \ldots, g_{n-1}$ declared in some classes $M_{0}, \ldots, M_{n-1}(n \geqslant 0)$. That is, for each $j: 0 \leqslant$ $j<n, X . g_{j} \in \operatorname{Comp}_{M_{j}}(X)$. For convenience, let $M_{n}$ be a synonym for $F$; then, $M_{0}$ is $T$. Now, the precondition of the update statement implies $\neg\left(x\right.$.inv $\left.<: M_{n}\right)$, so by $n$ applications of (4) and (6), we have $\neg\left(o . i n v<: M_{0}\right)$. In more detail, each of those $n$ applications goes as follows, for any $j$ : from $\neg\left(o . g_{0} \cdots \cdot g_{j+1}\right.$.inv $\left.<: M_{j+1}\right)$, static type checking, and (4), we have $\neg o . g_{0} \cdot \cdots \cdot g_{j+1}$. committed, and from that, the fact that $o . g_{0} \cdots \cdot g_{j+1}$ is not null, and (6) instantiated with $o:=o . g_{0} \cdot \cdots \cdot g_{j}$, we get $\neg\left(o . g_{0} \cdot \cdots \cdot g_{j}\right.$. inv $<: M_{j}$. This shows that (5) holds for $o$ in the post-state of the update statement, proving that program invariant (5) is maintained by the update statement. 
Finally, a field update statement $x . f:=E$ can change the value of an expression $p$ in $\mathrm{Comp}_{T}(x)$ only if $f$ is rep field declared in class $T$. But the precondition of the update statement implies $\neg(x$.inv $<: T)$, so the antecedent of both (6) and (7) is false. Hence, the invariance of (6) and (7) is maintained.

(End of Proof.)

\section{REFERENCES}

[0] Paulo Sérgio Almeida. Balloon types: Controlling sharing of state in data types. In Mehmet Aksit and Satoshi Matsuoka, editors, ECOOP'97 - Object-Oriented Programming, 11th European Conference, volume 1241 of Lecture Notes in Computer Science, pages 32-59. Springer, 1997.

[1] Anindya Banerjee and David A. Naumann. Ownership confinement ensures representation independence for object-oriented programs. Manuscript available on http://guinness.cs.stevens-tech.edu/ naumann/publications/, December 2002.

[2] Mike Barnett, Robert DeLine, Manuel Fähndrich, K. Rustan M. Leino, and Wolfram Schulte. Verification of object-oriented programs with invariants. In Susan Eisenbach, Gary T. Leavens, Peter Müller, Arnd Poetzsch-Heffter, and Erik Poll, editors, Formal Techniques for Java-like Programs 2003 (Proceedings), pages 61-68. Technical report 408, Department of Computer Science, ETH Zurich, July 2003.

[3] Mike Barnett and David Naumann. Friends need a bit more: Maintaining invariants over shared state. In Mathematics of Program Construction, Lecture Notes in Computer Science. Springer, July 2004. To appear.

[4] Boris Bokowski and Jan Vitek. Confined types. In Proceedings of the 1999 ACM SIGPLAN Conference on Object-Oriented Programming Systems, Languages and Applications (OOPSLA '99), volume 34, number 10 in SIGPLAN Notices, pages 82-96. ACM, October 1999.

[5] Chandrasekhar Boyapati, Robert Lee, and Martin Rinard. Ownership types for safe programming: Preventing data races and deadlocks. In Proceedings of the 2002 ACM SIGPLAN Conference on Object-Oriented Programming Systems, Languages and Applications, OOPSLA 2002, volume 37, number 11 in SIGPLAN Notices, pages 211-230. ACM, November 2002.

[6] John Boyland. Alias burying: Unique variables without destructive reads. Software-Practice \& Experience, 31(6):533-553, May 2001.

[7] Lilian Burdy, Yoonsik Cheon, David Cok, Michael D. Ernst, Joe Kiniry, Gary T. Leavens, K. Rustan M. Leino, and Erik Poll. An overview of JML tools and applications. In Thomas Arts and Wan Fokkink, editors, Eighth International Workshop 
on Formal Methods for Industrial Critical Systems (FMICS'03), volume 80 of Electronic Notes in Theoretical Computer Science. Elsevier, 2003.

[8] Dave G. Clarke and Tobias Wrigstad. External uniqueness is unique enough. In Luca Cardelli, editor, ECOOP 2003 - Object-Oriented Programming, 17th European Conference, volume 2743 of Lecture Notes in Computer Science, pages 176-200. Springer, 2003.

[9] David G. Clarke, John M. Potter, and James Noble. Ownership types for flexible alias protection. In Proceedings of the 1998 ACM SIGPLAN Conference on ObjectOriented Programming, Systems, Languages, and Applications (OOPSLA '98), volume 33, number 10 in SIGPLAN Notices, pages 48-64. ACM, October 1998.

[10] Robert DeLine and Manuel Fähndrich. Enforcing high-level protocols in low-level software. In Proceedings of the 2001 ACM SIGPLAN Conference on Programming Language Design and Implementation (PLDI), volume 36, number 5 in SIGPLAN Notices, pages 59-69. ACM, May 2001.

[11] Robert DeLine and Manuel Fähndrich. The Fugue protocol checker: Is your software Baroque? Technical Report MSR-TR-2004-07, Microsoft Research, January 2004.

[12] Robert DeLine and Manuel Fähndrich. Typestates for objects. In ECOOP 2004 Object-Oriented Programming, 18th European Conference, June 2004. To appear.

[13] David L. Detlefs, K. Rustan M. Leino, and Greg Nelson. Wrestling with rep exposure. Research Report 156, Digital Equipment Corporation Systems Research Center, July 1998.

[14] David L. Detlefs, K. Rustan M. Leino, Greg Nelson, and James B. Saxe. Extended static checking. Research Report 159, Compaq Systems Research Center, December 1998.

[15] Manuel Fähndrich and Robert DeLine. Adoption and focus: Practical linear types for imperative programming. In Proceedings of the 2002 ACM SIGPLAN Conference on Programming Language Design and Implementation (PLDI), volume 37, number 5 in SIGPLAN Notices, pages 13-24. ACM, May 2002.

[16] Cormac Flanagan, K. Rustan M. Leino, Mark Lillibridge, Greg Nelson, James B. Saxe, and Raymie Stata. Extended static checking for Java. In Proceedings of the 2002 ACM SIGPLAN Conference on Programming Language Design and Implementation (PLDI), volume 37, number 5 in SIGPLAN Notices, pages 234-245. ACM, May 2002.

[17] Aaron Greenhouse and John Boyland. An object-oriented effects system. In Rachid Guerraoui, editor, ECOOP'99 - Object-Oriented Programming, 13th European Conference, volume 1628 of Lecture Notes in Computer Science, pages 205-229. Springer, June 1999. 
[18] John V. Guttag and James J. Horning, editors. Larch: Languages and Tools for Formal Specification. Texts and Monographs in Computer Science. Springer-Verlag, 1993. With Stephen J. Garland, Kevin D. Jones, Andrés Modet, and Jeannette M. Wing.

[19] C. A. R. Hoare. Proof of correctness of data representations. Acta Informatica, 1(4):271-81, 1972.

[20] C. A. R. Hoare. Monitors: an operating system structuring concept. Communications of the ACM, 17(10):549-557, October 1974.

[21] John Hogg. Islands: Aliasing protection in object-oriented languages. In Proceedings of the sixth ACM SIGPLAN Conference on Object-Oriented Programming, Systems, Languages, and Applications (OOPSLA '91), 1991.

[22] Kees Huizing and Ruurd Kuiper. Verification of object-oriented programs using class invariants. In Tom Maibaum, editor, Fundamental Approaches to Software Engineering, Third International Conference, FASE 2000, volume 1783 of Lecture Notes in Computer Science, pages 208-221. Springer, 2000.

[23] Samin Ishtiaq and Peter W. O'Hearn. BI as an assertion language for mutable data structures. In Symposium on Principles of Programming Languages, pages 14-26, 2001.

[24] Gary T. Leavens, Albert L. Baker, and Clyde Ruby. JML: A notation for detailed design. In Haim Kilov, Bernhard Rumpe, and Ian Simmonds, editors, Behavioral Specifications of Businesses and Systems, pages 175-188. Kluwer Academic Publishers, 1999.

[25] K. Rustan M. Leino. Toward Reliable Modular Programs. PhD thesis, California Institute of Technology, 1995. Technical Report Caltech-CS-TR-95-03.

[26] K. Rustan M. Leino. Data groups: Specifying the modification of extended state. In Proceedings of the 1998 ACM SIGPLAN Conference on Object-Oriented Programming, Systems, Languages, and Applications (OOPSLA '98), volume 33, number 10 in SIGPLAN Notices, pages 144-153. ACM, October 1998.

[27] K. Rustan M. Leino and Peter Müller. Object invariants in dynamic contexts. In ECOOP 2004 - Object-Oriented Programming, 18th European Conference, June 2004. To appear.

[28] K. Rustan M. Leino and Greg Nelson. Data abstraction and information hiding. ACM Transactions on Programming Languages and Systems, 24(5):491-553, September 2002.

[29] K. Rustan M. Leino, Greg Nelson, and James B. Saxe. ESC/Java user's manual. Technical Note 2000-002, Compaq Systems Research Center, October 2000. 
[30] K. Rustan M. Leino, Arnd Poetzsch-Heffter, and Yunhong Zhou. Using data groups to specify and check side effects. In Proceedings of the 2002 ACM SIGPLAN Conference on Programming Language Design and Implementation (PLDI), pages 246257, 2002.

[31] K. Rustan M. Leino and Raymie Stata. Checking object invariants. Technical Note 1997-007, Digital Equipment Corporation Systems Research Center, January 1997.

[32] Barbara Liskov and John Guttag. Abstraction and Specification in Program Development. MIT Electrical Engineering and Computer Science Series. MIT Press, 1986.

[33] Bertrand Meyer. Object-oriented software construction. Series in Computer Science. Prentice-Hall International, 1988.

[34] Naftaly H. Minsky. Towards alias-free pointers. In Pierre Cointe, editor, ECOOP'96 - Object-Oriented Programming, 10th European Conference, volume 1098 of Lecture Notes in Computer Science, pages 189-209. Springer, July 1996.

[35] Peter Müller. Modular Specification and Verification of Object-Oriented Programs, volume 2262 of Lecture Notes in Computer Science. Springer-Verlag, 2002. PhD thesis, FernUniversität Hagen.

[36] Peter Müller and Arnd Poetzsch-Heffter. Universes: A type system for alias and dependency control. Technical Report 279, FernUniversität Hagen, 2001.

[37] Peter Müller, Arnd Poetzsch-Heffter, and Gary T. Leavens. Modular invariants for object structures. Technical Report 424, ETH Zurich, October 2003.

[38] James Noble, Jan Vitek, and John Potter. Flexible alias protection. In Eric Jul, editor, ECOOP'98 - Object-Oriented Programming, 12th European Conference, volume 1445 of Lecture Notes in Computer Science, pages 158-185. Springer, July 1998.

[39] Arnd Poetzsch-Heffter. Specification and verification of object-oriented programs. Habilitationsschrift, Technische Universität München, 1997.

[40] David Walker and Greg Morrisett. Alias types for recursive data structures. In Proceedings of the 4th Workshop on Types in Compilation, September 2000.

[41] Alan Cameron Wills. Formal Methods applied to Object-Oriented Programming. $\mathrm{PhD}$ thesis, University of Manchester, Department of Computer Science, 1992. 


\section{ABOUT THE AUTHORS}
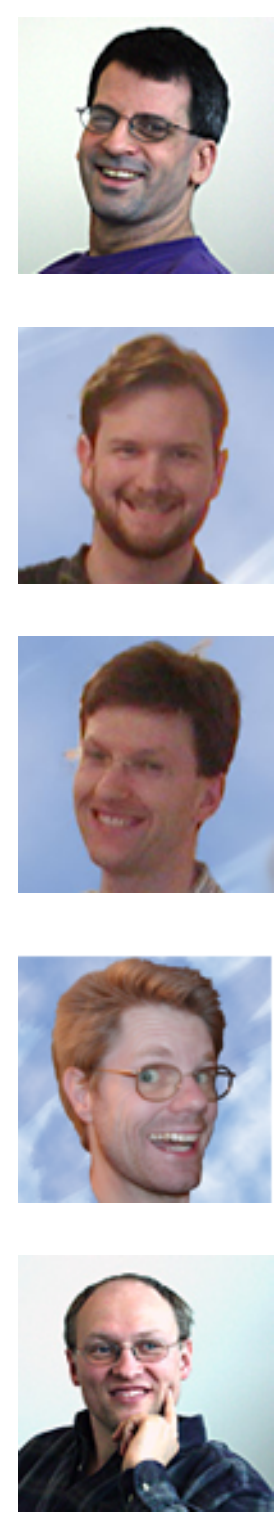

Mike Barnett is a strong supporter of esoteric radio stations. That's how he learns about the latest hip music before his teenagers do.

Robert DeLine is a gifted carpenter. He is known for his carefully constructed multi-level staircases.

Manuel Fähndrich is an architect of backyard dwellings for minors. Combining fun and safety, his creations stand up to any weather.

K. Rustan M. Leino is a starving musician. In order to support himself, he has a wife and four working children.

Wolfram Schulte is an avid bicyclist. Every day, he rides toward a destination uphill from his home. 